\title{
Star formation associated with a large-scale infrared bubble ${ }^{\star}$
}

\author{
Jin-Long $\mathrm{Xu}^{1,2}$ and Bing-Gang $\mathrm{Ju}^{3,4}$ \\ ${ }^{1}$ National Astronomical Observatories, Chinese Academy of Sciences, Beijing 100012, PR China \\ e-mail: xujl@bao.ac.cn \\ 2 NAOC-TU Joint Center for Astrophysics, Lhasa 850000, PR China \\ 3 Purple Mountain Observatory, Qinghai Station, 817000, Delingha, PR China \\ ${ }^{4}$ Key Laboratory of Radio Astronomy, Chinese Academy of Sciences, Nanjing 210008, PR China
}

Received 7 April 2014 / Accepted 16 July 2014

\section{ABSTRACT}

\begin{abstract}
Aims. To investigate how a large-scale infrared bubble centered at $l=53.9^{\circ}$ and $b=0.2^{\circ}$ forms, and to study if star formation is taking place at the periphery of the bubble, we performed a multiwavelength study.

Methods. Using the data from the Galactic Ring Survey (GRS) and Galactic Legacy Infrared Mid-Plane Survey Extraordinaire (GLIMPSE), we performed a study of a large-scale infrared bubble with a size of about $16 \mathrm{pc}$ at a distance of $2.0 \mathrm{kpc}$. We present the ${ }^{12} \mathrm{CO} J=1-0,{ }^{13} \mathrm{CO} J=1-0$, and $\mathrm{C}^{18} \mathrm{O} J=1-0$ observations of HII region G53.54-0.01 (Sh2-82) obtained at the Purple Mountain Observation (PMO) $13.7 \mathrm{~m}$ radio telescope to investigate the detailed distribution of associated molecular material. In addition, we also used radiorecombination line and VLA data. To select young stellar objects (YSOs) consistent with this region, we used the GLIMPSE I catalog.

Results. The large-scale infrared bubble shows a half-shell morphology at $8 \mu \mathrm{m}$. The H II regions of G53.54-0.01, G53.64+0.24, and G54.09-0.06 are situated on the bubble. Comparing the radio recombination line velocities and associated ${ }^{13} \mathrm{CO} J=1-0$ components of the three H II regions, we found that the $8 \mu \mathrm{m}$ emission associated with H II region G53.54-0.01 should belong to the foreground emission, and only overlap with the large-scale infrared bubble in the line of sight. Three extended green objects (EGOs, the candidate massive young stellar objects), as well as three H II regions and two small-scale bubbles are found located in the G54.09-0.06 complex, indicating an active massive star-forming region. Emission from $\mathrm{C}^{18} \mathrm{O}$ at $J=1-0$ presents four cloud clumps on the northeastern border of $\mathrm{H}$ II region G53.54-0.01. By comparing the spectral profiles of ${ }^{12} \mathrm{CO} J=1-0,{ }^{13} \mathrm{CO} J=1-0$, and $\mathrm{C}^{18} \mathrm{O} J=1-0$ at the peak position of each clump, we found the collected gas in the three clumps, except for the clump coinciding with a massive YSO (IRAS 19282+1814). Using the evolutive model of the H II region, we derived that the age of H II region G53.54-0.01 is $1.5 \times 10^{6} \mathrm{yr}$. The significant enhancement of several Class I and Class II YSOs around G53.54-0.01 indicates the presence of some recently formed stars, which may be triggered by this H II region through the collect-and-collapse process.
\end{abstract}

Key words. stars: early-type - HII regions - ISM: individual objects: Sh2-82 - ISM: individual objects: G54.09-0.06 stars: formation

\section{Introduction}

Star formation requires dense self-gravitating gas. The activity of ultraviolet (UV) radiation, stellar winds, and supernova remnants (SNRs) can all compress or accumulate a pre-existing gas into a dense gas. The gas may show gravitational instabilities, then collapse into dense cores. These three dynamic processes may trigger the formation of a new generation of stars. Churchwell et al. $(2006,2007)$ compiled a list of $\sim 600$ objects with $8 \mu \mathrm{m}$ emission in a bubble morphology, suggesting that the bubbles were polycyclic aromatic hydrocarbon (PAH) emission in the photodissociation regions (PDRs) surrounding $\mathrm{O}$ and early-B stars. Deharveng et al. (2010) studied 102 bubbles cataloged by Churchwell et al. (2006, 2007), most of which enclose $\mathrm{H}$ II regions ionized by O-B2 stars. They also concluded that the bubbles may trigger $14 \% \sim 30 \%$ of the star formation in our Galaxy. Recently several pieces of observational evidence have been found in favor of star formation triggered by bubbles (see,

* Final CO cubes $(12,13,18$, FITS format) are only available at the CDS via anonymous ftp to cdsarc.u-strasbg. fr (130.79.128.5) or via

http://cdsarc.u-strasbg.fr/viz-bin/qcat?]/A+A/569/A36 e.g., Dewangan et al. 2012; Ji et al. 2012; Zhang et al. 2013; Dewangan \& Ojha 2013; Li et al. 2014).

Leahy et al. (2008) identified a large-scale infrared bubble at $8 \mu \mathrm{m}$ from the Galactic Legacy Infrared Mid-Plane Survey Extraordinaire (GLIMPSE) project, which just surrounds a large radio shell (centered at $l=53.9^{\circ}$ and $b=0.2^{\circ}$ ) with an angular size of $30^{\prime} \times 26^{\prime}$. Velusamy et al. (1986) classified the radio shell as an $\mathrm{H}$ II region because of possible thermal infrared emission. With a higher spatial resolution, Leahy et al. (2008) showed that the radio shell and infrared bubble are physically separated, suggesting that the radio shell may be an old SNR at a distance of $\simeq 7.3 \mathrm{kpc}$. There are three $\mathrm{H}$ II regions (G53.54-0.01, G53.64+0.24, and G54.09-0.06) and a young SNR (G54.1+0.3) in the infrared bubble, as seen in Fig. 1.

The H II region G53.54-0.01(Sh2-82) coincides with the bubble N115 (Churchwell et al. 2006; Deharveng et al. 2010; Sherman 2012). Hunter \& Massey (1990) gave that HD 231616 with a B0V type and a mass of $18 M_{\odot}$ may be the exciting star of G53.54-0.01. Using the Galactic Ring Survey (GRS) data, two clumps of ${ }^{13} \mathrm{CO} J=1-0$ were identified at the northeast of G53.54-0.01 (Yu et al. 2012). The source G54.1+0.3 is a corecollapse SNR with centrally brightened synchrotron emission in radio and X-rays (Velusamy \& Becker 1988; Lu et al. 2002), 


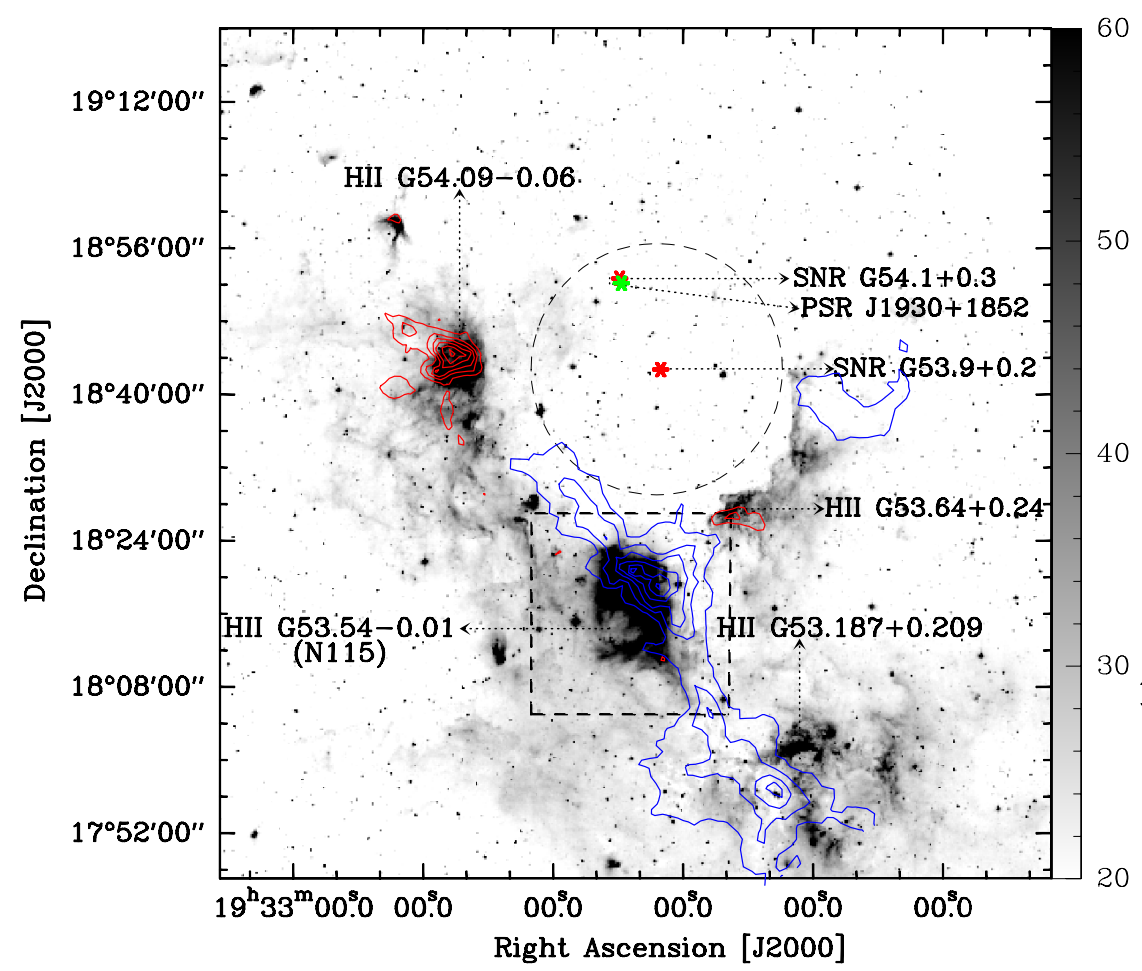

50

40

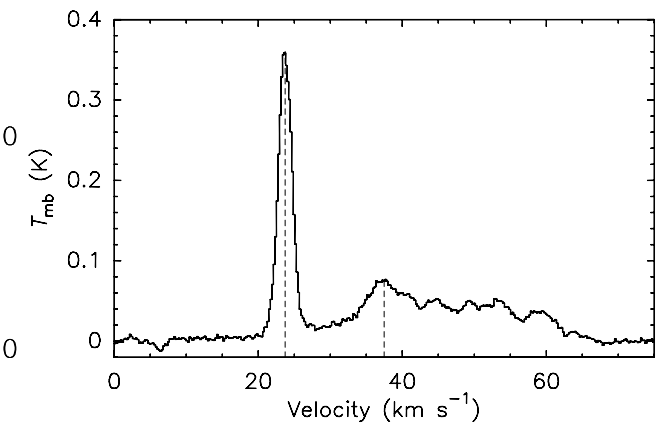

Fig. 1. Left panel: ${ }^{13} \mathrm{CO} J=1-0$ integrated intensity contours (blue and red) overlayed on the Spitzer-IRAC $8 \mu \mathrm{m}$ emission map (gray scale). The blue contour levels are $20,35, \ldots, 95 \%$ of the peak value $\left(19.9 \mathrm{~K} \mathrm{~km} \mathrm{~s}^{-1}\right)$ integrated from $20-28 \mathrm{~km} \mathrm{~s}^{-1}$, while the red contour levels are 20,35 , ..., $95 \%$ of the peak value $\left(26.1 \mathrm{~K} \mathrm{~km} \mathrm{~s}^{-1}\right)$ integrated from $35-43 \mathrm{~km} \mathrm{~s}^{-1}$. The different sources associated with the region are marked. The red and green stars represent the center of SNRs and PSR. The radius of the radio shell is $14^{\prime}$ outlined by a dashed circle. The dashed square frame marks the area observed with PMO telescope. The gray bar is in units of $\mathrm{MJy} \mathrm{sr}^{-1}$. Right panel: average spectra of ${ }^{13} \mathrm{CO} J=1-0$ over the whole large-scale infrared bubble. The vertical dashed lines in the spectra mark the peak velocity.

which closely resembles the Crab Nebula. By the $\mathrm{HI}$ and $\mathrm{CO}$ observations, Leahy et al. (2008) gave the kinematic distance of $\simeq 6.2 \mathrm{kpc}$ to G54.1+0.3. The SNR is associated with a pulsar (PSR J1930+1852), which has a $136 \mathrm{~ms}$ rotational period and a characteristic age of 2900 yr (Camilo et al. 2002; Lu et al. 2008). Koo et al. (2008) detected a star-forming loop around SNR G54.1+0.3 using the AKARI infrared satellite. They proposed that the star-forming loop is triggered by the progenitor star of G54.1+0.3.

To explore signatures of star formation in a large-scale infrared bubble, we mainly combined molecular, infrared, and radio continuum observations toward H II regions G53.54-0.01 and G54.09-0.06. The observations and data reduction are described in Sect. 2, and the results are presented in Sect. 3. In Sect. 4, we discuss how our data provide evidence of triggered star formation in the large-scale infrared bubble. The conclusions are summarized in Sect. 5.

\section{Observations and data reduction}

\section{1. ${ }^{13} \mathrm{CO} J=1-0 \mathrm{GRS}$ data}

The GRS is a survey of ${ }^{13} \mathrm{CO} J=1-0$ emission (Jackson et al. 2006). The survey covers a longitude range of $\ell=18^{\circ}-55.7^{\circ}$ and a latitude range of $|b| \leq 1^{\circ}$, with a angular resolution of $46^{\prime \prime}$. The survey's velocity coverage is $-5 \mathrm{~km} \mathrm{~s}^{-1}$ to $135 \mathrm{~km} \mathrm{~s}^{-1}$ for Galactic longitudes $\ell \leq 40^{\circ}$ and $-5 \mathrm{~km} \mathrm{~s}^{-1}$ to $85 \mathrm{~km} \mathrm{~s}^{-1}$ for Galactic longitudes $\ell>40^{\circ}$. At the velocity resolution of $0.21 \mathrm{~km} \mathrm{~s}^{-1}$, the typical $\mathrm{rms}$ sensitivity is $0.13 \mathrm{~K}$. We used the
GRS archival data ${ }^{1}$ to study the molecular emission of the largescale infrared bubble.

\subsection{Purple Mountain data}

The mapping observations of $\mathrm{H}$ II region G53.54-0.01 and its adjacent region were performed in the ${ }^{12} \mathrm{CO} J=1-0$, ${ }^{13} \mathrm{CO} J=1-0$, and $\mathrm{C}^{18} \mathrm{O} J=1-0$ lines using the Purple Mountain Observation (PMO) $13.7 \mathrm{~m}$ radio telescope (Zuo et al. 2004) at De Ling $\mathrm{Ha}$ in the west of China at an altitude of 3200 meters, in May 2013. The Superconducting Spectroscopic Array Receiver (SSAR) is used, which is a superconducting focal plane array that observes a $3 \times 3$ beam. The new array receiver system in single-sideband (SSB) mode was used as front end. The back end is a fast Fourier transform spectrometer (FFTS) of 16384 channels with a bandwidth of $1 \mathrm{GHz}$, corresponding to a velocity resolution of $0.16 \mathrm{~km} \mathrm{~s}^{-1}$ for ${ }^{12} \mathrm{CO} J=1-0$ and $0.17 \mathrm{~km} \mathrm{~s}^{-1}$ for ${ }^{13} \mathrm{CO} J=1-0$ and $\mathrm{C}^{18} \mathrm{O} J=1-0 ;{ }^{12} \mathrm{CO} J=1-0$ was observed at upper sideband, while ${ }^{13} \mathrm{CO} J=1-0$ and $\mathrm{C}^{18} \mathrm{O} J=1-0$ were observed simultaneously at lower sideband. The half-power beam width (HPBW) was 53" at $115 \mathrm{GHz}$ and the main beam efficiency was 0.5 . The pointing accuracy of the telescope was better than 5 ", which was derived from continuum observations of planets. The source W51D was observed once per hour as flux calibrator. The system noise temperature (Tsys) in SSB mode varied between $150 \mathrm{~K}$ and $400 \mathrm{~K}$. Mapping observations were centered at $\mathrm{RA}(\mathrm{J} 2000)=19^{\mathrm{h}} 30^{\mathrm{m}} 23.98^{\mathrm{s}}, \operatorname{Dec}(\mathrm{J} 2000)=18^{\circ} 15^{\prime} 03.3^{\prime \prime}$ using on-the-fly (OTF) observing mode. The total mapping area

1 http://www.bu.edu/galacticring/ 


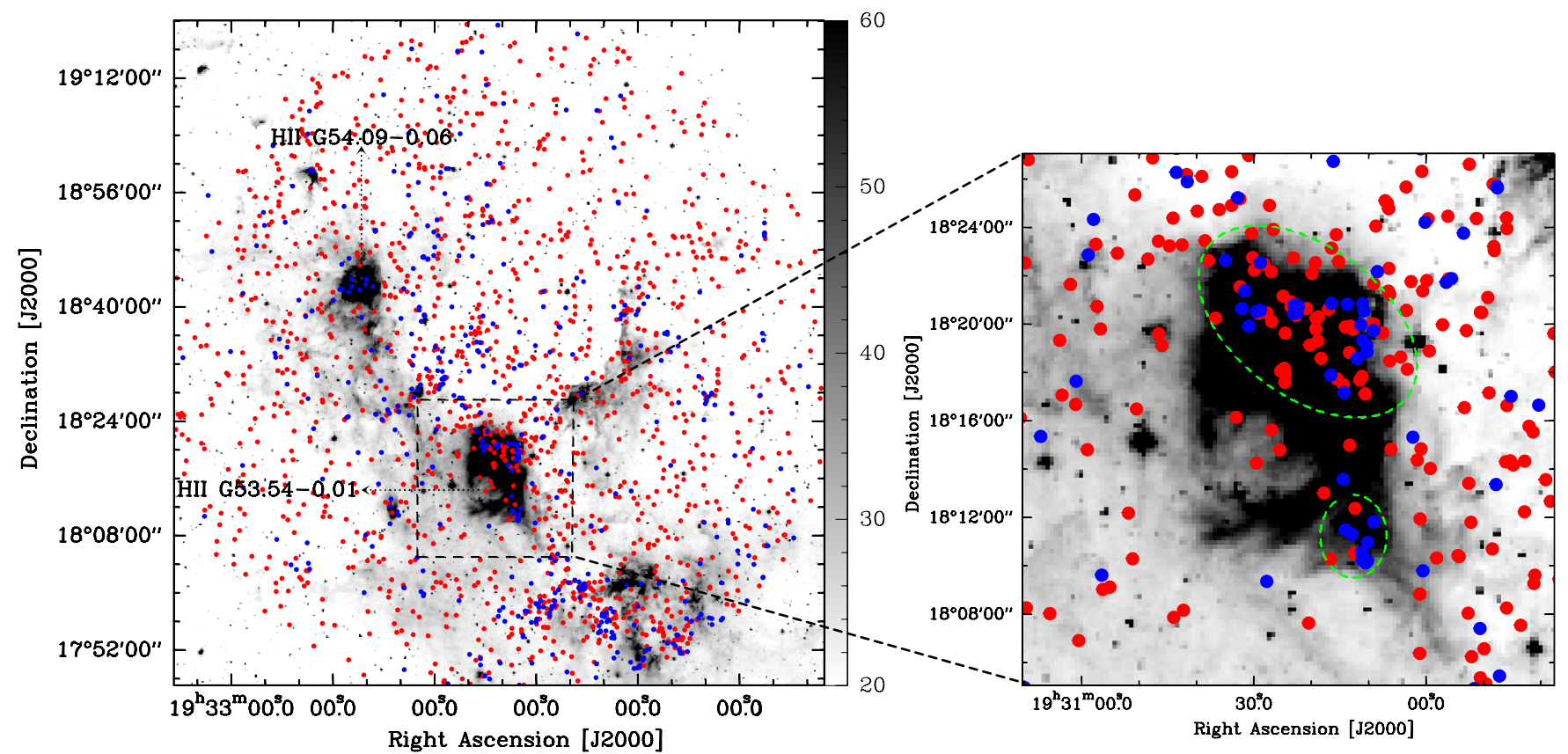

Fig. 2. Left panel: Spitzer-IRAC $8 \mu \mathrm{m}$ emission map (gray scale) overlayed on Class I and Class II sources labeled as the blue and red dots, respectively. The unit of the gray bar is in $\mathrm{MJy} \mathrm{sr}^{-1}$. Right panel: distributed map of Class I and Class II sources in the vicinity of $\mathrm{H}$ II region G53.54-0.01. Two green dashed ellipses mark the concentration of Class I and Class II sources.

is $15^{\prime} \times 15^{\prime}$ in ${ }^{12} \mathrm{CO} J=1-0,{ }^{13} \mathrm{CO} J=1-0$, and $\mathrm{C}^{18} \mathrm{O} J=1-0$ with a $0.5^{\prime} \times 0.5^{\prime}$ grid. The standard chopper wheel calibration technique was used to measure antenna temperature $T_{\mathrm{A}}^{*}$ corrected for atmospheric absorption. The final data was recorded in brightness temperature scale of $T_{\mathrm{mb}}(\mathrm{K})$. The data were reduced using the GILDAS/CLASS ${ }^{2}$ package.

\subsection{Additional data}

The $1.4 \mathrm{GHz}$ radio continuum emission data were obtained from the NRAO VLA Sky Survey (NVSS; Condon et al. 1998); NVSS is a $1.4 \mathrm{GHz}$ continuum survey covering the entire sky north of $-40^{\circ}$ declination (1998) with a noise of about $0.45 \mathrm{mJy} / \mathrm{beam}$ and the resolution of $45^{\prime \prime}$.

The radio-recombination lines data were derived from the GBT survey (Anderson et al. 2011). The survey has detected 448 previously unknown Galactic $\mathrm{H}$ II regions at X-band $(9 \mathrm{GHz}$, $3 \mathrm{~cm}$ ) in the Galactic zone $343^{\circ} \leq \ell \leq 67^{\circ}$ and $|b| \leq 1^{\circ}$. The FWHM beam size of the telescope is approximately $82^{\prime \prime}$ at this frequency.

The GLIMPSE survey was used to identify the young stars along H II regions, which observed the Galactic plane $\left(65^{\circ}<\right.$ $|l|<10^{\circ}$ for $|b|<1^{\circ}$ ) with the four IR bands (3.6, 4.5, 5.8, and $8.0 \mu \mathrm{m}$ ) of the Infrared Array Camera (IRAC; Fazio et al. 2004) on the Spitzer Space Telescope. The resolution is from $1.5^{\prime \prime}(3.6 \mu \mathrm{m})$ to $1.9^{\prime \prime}(8.0 \mu \mathrm{m})$.

\section{Results}

\subsection{The large-scale infrared bubble}

\subsubsection{Infrared and ${ }^{13} \mathrm{CO} J=1-0$ emission}

Figure 1, left panel shows the ${ }^{13} \mathrm{CO} J=1-0$ integrated intensity map superimposed on the Spitzer-IRAC emission at $8 \mu \mathrm{m}$ (gray

\footnotetext{
2 http://www.iram.fr/IRAMFR/GILDAS/
}

scale). The Spitzer-IRAC $8 \mu \mathrm{m}$ emission presents a half-shell morphology corresponding mainly to fluorescence from $\mathrm{PAH}$ molecules. The density of the inner edge is higher in the largescale infrared bubble. According to Leahy et al. (2008), the infrared bubble around a large radio shell with an angular size of $30^{\prime} \times 26^{\prime}$ contains three $\mathrm{H}$ II regions. Two extended H II regions G54.09-0.06 and G53.64+0.24 have recombination line velocities of $42.1 \pm 0.8 \mathrm{~km} \mathrm{~s}^{-1}$ and $38 \pm 1.8 \mathrm{~km} \mathrm{~s}^{-1}$, respectively. The recombination line velocity of $\mathrm{H}$ II region G53.54-0.01 is $23.9 \pm 0.3 \mathrm{~km} \mathrm{~s}^{-1}$ (Anderson et al. 2011).

The CO maps contain velocity information that allows us to disentangle different molecular components along the line of sight. The right panel of Fig. 2 shows the average spectra of ${ }^{13} \mathrm{CO} J=1-0$ over the whole large-scale infrared bubble. From the spectra, we can see that there is a strong line in the velocity intervals from $20 \mathrm{~km} \mathrm{~s}^{-1}$ to $28 \mathrm{~km} \mathrm{~s}^{-1}$ with a peak at $\sim 24 \mathrm{~km} \mathrm{~s}^{-1}$. Other velocity components overlap between 30 and $63 \mathrm{~km} \mathrm{~s}^{-1}$. In the overlapping range of velocity, there is a relatively strong line located in the velocity intervals $35 \mathrm{~km} \mathrm{~s}^{-1}$ to $43 \mathrm{~km} \mathrm{~s}^{-1}$ peak at $\sim 38 \mathrm{~km} \mathrm{~s}^{-1}$. Using the velocity ranges of 20-28 $\mathrm{km} \mathrm{s}^{-1}$ and $35-43 \mathrm{~km} \mathrm{~s}^{-1}$, we made the integrated intensity map of ${ }^{13} \mathrm{CO} J=1-0$ (Fig. 1, right panel), marked by red and blue contours, respectively. In Fig. 1, the velocity component of $20-28 \mathrm{~km} \mathrm{~s}^{-1}$ presents a filamentary structure, which is associated well with the infrared emission of $\mathrm{H}$ II region G53.54-0.01. However, the filamentary structure extends toward the center of the large-scale infrared bubble. A weak component of 20 to $28 \mathrm{~km} \mathrm{~s}^{-1}$ is located on the western border of the infrared bubble. Additionally, the velocity components in the range of $35-43 \mathrm{~km} \mathrm{~s}^{-1}$ are consistent with the infrared emission of $\mathrm{H}$ II regions G54.09.0.06 and G53.64+0.24. The ${ }^{13} \mathrm{CO} J=$ 1-0 emission related to the H II region G54.09.0.06 shows a comet-like morphology. Hence, the large-scale infrared bubble may consist of two components, while the velocity component in the interval $20-28 \mathrm{~km} \mathrm{~s}^{-1}$ should belong to the foreground emission, and only overlap with the other component in the line of sight. 


\subsubsection{Search for young stellar objects}

To study star formation in the vicinity of the large-scale infrared bubble by detecting all the young stellar objects (YSOs) around the infrared bubble and looking at their position with respect to the ionized gas and molecular condensations, we used the Spitzer-GLIMPSE I catalog. Considering only sources that have been detected in the four Spitzer-IRAC bands, we found 153286 near-infrared sources centered on the infrared shell within a circle of $48^{\prime}$ in radius. Allen et al. (2004) showed that YSOs have specific infrared colors depending on their masses and their evolutionary stages. Based on the color selection criteria of YSOs (Allen et al. 2004), we found 478 Class I sources and 1423 Class II sources. Class I sources are protostars with circumstellar envelopes with an age of $\sim 10^{5} \mathrm{yr}$, while Class II sources are diskdominated objects with an age of $\sim 10^{6} \mathrm{yr}$. The left panel of Fig. 2 presents the spatial distribution of Class I and Class II sources. From the left panel, we see that Class I and Class II sources are symmetrically distributed across the whole selected region, but concentrated around G53.54-0.01. In order to clearly show the distribution of YSOs in the vicinity of H II region G53.54-0.01, we made a small-scale distributed map of the YSOs shown in the right panel of Fig. 2, which obviously presents the concentration of YSOs at the border of G53.54-0.01. We do not know if all the YSOs seen in the direction of G53.54-0.01 lie at the same distance as with the H II region and are associated with it. However, the concentration of YSOs in the surroundings of G53.54-0.01 indicates that the association is highly probable.

\section{2. $H$ II region G54.09-0.06}

In Fig. 3, the $1.4 \mathrm{GHz}$ continuum emission obtained from NVSS exhibits an extended structure, which is consistent with the Spitzer-IRAC $8 \mu \mathrm{m}$ emission. From the survey of H II regions at the radio band in the northern sky (Lockman 1989), we found two other H II regions (G50.10-0.06 and G54.1-0.1) that may be associated with the Spitzer-IRAC $8 \mu \mathrm{m}$ emission. However, H II region G50.10-0.06 is closer to the center of the $1.4 \mathrm{GHz}$ continuum emission. IRAS $19294+1836$ with a velocity of $39.9 \mathrm{~km} \mathrm{~s}^{-1}$ is a YSO, which is nearer to at the position of G54.09-0.06. Additionally, there are three extended green objects (EGOs) (G54.11-0.04, 54.11-0.05, and 54.11-0.08) in the region; EGOs have excess emission in extended structures in the Infrared Array Camera (IRAC) $4.5 \mu \mathrm{m}$ band images that are conventionally coded as green in the IRAC false color images, which are considered as the candidate birth places of the massive YSO (Cyganowski et al. 2008). He et al. (2012) found that the velocity of EGO $54.11-0.08$ is $38.4 \mathrm{~km} \mathrm{~s}^{-1}$, while that of EGO G54.11-0.04 is $39.2 \mathrm{~km} \mathrm{~s}^{-1}$ (Chen et al. 2010), suggesting that this region is is the site of formation of many massive stars. Moreover, two small-scale bubbles (N116 and N117) are located in this region, selected from the catalog of Churchwell et al. (2006) bubbles. Watson et al. (2010) found that the velocity of $\mathrm{N} 117$ is $18.7 \mathrm{~km} \mathrm{~s}^{-1}$ from the survey of $\mathrm{H}$ II regions in the northern sky (Lockman 1989), but we did not find the matching source with N117 in the survey results. As shown in Fig. 1 (right panel), the comet-like component of ${ }^{13} \mathrm{CO} J=1-0$ with a peak at $38 \mathrm{~km} \mathrm{~s}^{-1}$ presents a good morphological correlation with the infrared emission containing G54.09.0.06. From Fig. 3, we also see that N116 and N117 are associated well with the SpitzerIRAC $8 \mu \mathrm{m}$ emission, suggesting that the velocity of N116 and $\mathrm{N} 117$ may be $\sim 38 \mathrm{~km} \mathrm{~s}^{-1}$.

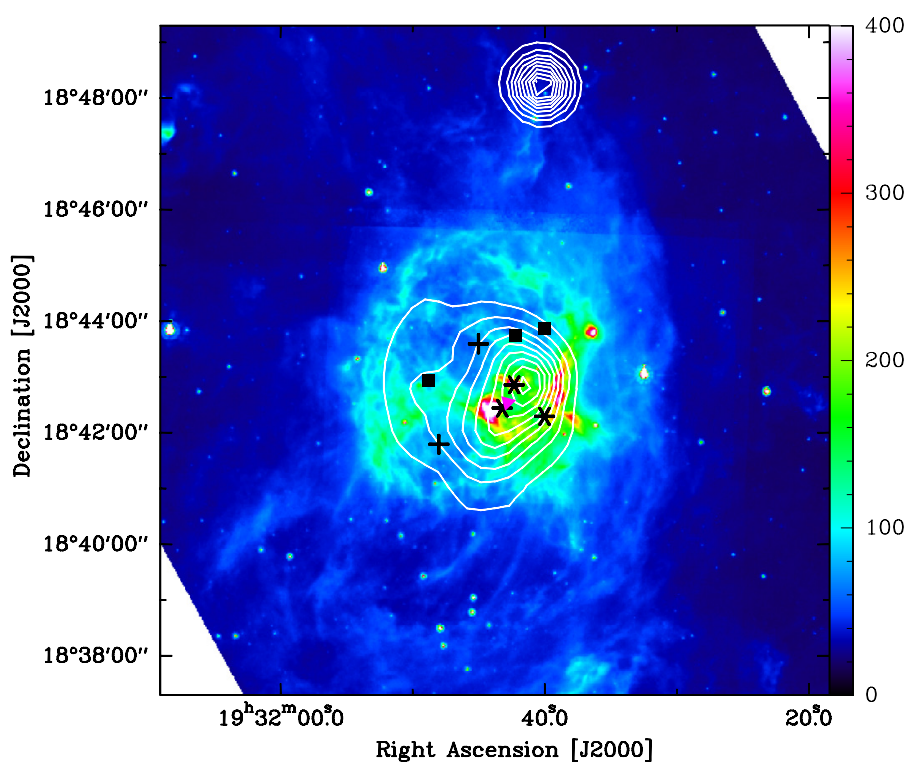

Fig. 3. 1.4 GHz radio continuum emission contours (white) overlayed on the Spitzer-IRAC $8 \mu \mathrm{m}$ emission map (color scale). The white contour levels are $20,35, \ldots, 95 \%$ of the peak value $\left(0.3 \mathrm{Jy}_{\text {beam }^{-1}}\right)$. Two black crosses indicate N116 and N117, and the "*" show three HII regions. The EGO sources are labeled as the filled square symbols. The pink triangle represents the IRAS $19294+1836$ source. The color bar is in units of $\mathrm{MJy} \mathrm{sr}^{-1}$

\section{3. $H$ II region G53.54-0.01}

The concentration of YSOs around H II region G53.54-0.01 indicates that their formation might have been triggered. The PAH emission of H II region G53.54-0.01 shows the cometary globule. To analyze in greater detail the morphology of molecular gas associated with G53.54-0.01, we mapped the emission in the ${ }^{12} \mathrm{CO} J=1-0,{ }^{13} \mathrm{CO} J=1-0$, and $\mathrm{C}^{18} \mathrm{O} J=1-0$ transitions. Adopting the velocity range of $20-28 \mathrm{~km} \mathrm{~s}^{-1}$ from the right panel of Fig. 1, the integrated intensity maps of ${ }^{13} \mathrm{CO} J=1-0$ and $\mathrm{C}^{18} \mathrm{O} J=1-0$ are shown in Fig. 4 a where we see that the emission of ${ }^{13} \mathrm{CO} J=1-0$ shows the arc-like structure consistent with the PAH emission to the northwest of G53.540.01 . Four clumps are identified in $\mathrm{C}^{18} \mathrm{O} J=1-0$, designated clumps A-D, respectively. There are two submillimeter wavelength continuum sources and an IRAS source that are associated with the molecular emission (Di Francesco et al. 2008 \& Sun et al. 2003). Moreover, JCMTSF J193024.2+182026 and IRAS $19282+1814$ are nearly located in the center of clump A. Sun et al. (2003) suggested that IRAS $19282+1814$ may be a massive YSO candidate.

Figure 5 shows the ${ }^{12} \mathrm{CO} J=1-0,{ }^{13} \mathrm{CO} J=1-0$, and $\mathrm{C}^{18} \mathrm{O}$ $J=1-0$ spectra toward the peak positions of each clump. The line profiles of ${ }^{12} \mathrm{CO} J=1-0$ and ${ }^{13} \mathrm{CO} J=1-0$ in clump A appear to only show the blue wings. For clump B, the line profiles of ${ }^{12} \mathrm{CO} J=1-0$ and ${ }^{13} \mathrm{CO} J=1-0$ are only broadened in the red wings, while that of $\mathrm{C}^{18} \mathrm{O} J=1-0$ shows double peaks. The line profiles of ${ }^{12} \mathrm{CO} J=1-0$ and ${ }^{13} \mathrm{CO} J=1-0$ in clumps $\mathrm{C}$ and $\mathrm{D}$ are the same as that in clump B, but both line profiles of $\mathrm{C}^{18} \mathrm{O} J=1-0$ show a single peak. The profile of each line is divided into a main and a residual part in the red wing. We fitted each spectrum with two Gaussian profiles. Table 1 shows the fitted results. Adopting two velocity ranges of $19-24 \mathrm{~km} \mathrm{~s}^{-1}$ and $24-27 \mathrm{~km} \mathrm{~s}^{-1}$, we also made the integrated intensify maps of ${ }^{12} \mathrm{CO} J=1-0,{ }^{13} \mathrm{CO} J=1-0$ and $\mathrm{C}^{18} \mathrm{O} J=1-0$ shown 
J.-L. Xu and B.-G. Ju: Star formation associated with a large-scale infrared bubble
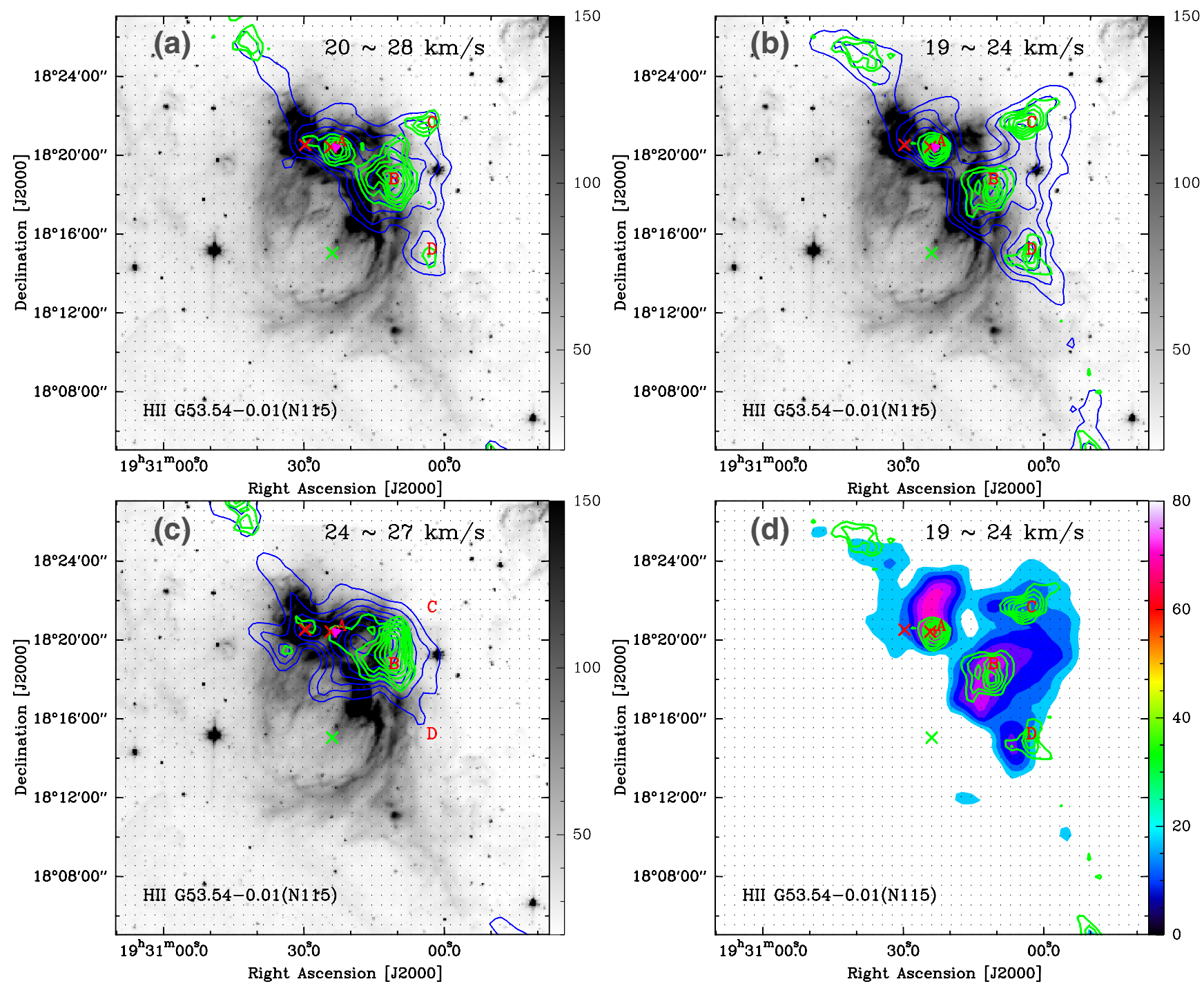

Fig. 4. a) Spitzer-IRAC $8 \mu \mathrm{m}$ emission map (gray scale) superimposed on ${ }^{13} \mathrm{CO} J=1-0$ (blue contours) and $\mathrm{C}^{18} \mathrm{O} J=1-0$ (green contours) intensity maps. The integrated velocity range is from $20-28 \mathrm{~km} \mathrm{~s}^{-1}$. b) Integrated velocity range is from $19-24 \mathrm{~km} \mathrm{~s}^{-1}$. c) Integrated velocity range is from $24-27 \mathrm{~km} \mathrm{~s}^{-1}$. d) $\mathrm{C}^{18} \mathrm{O} J=1-0$ (green contours) intensity map overlayed on ${ }^{12} \mathrm{CO} J=1-0$ integrated intensity map (color scale). The integrated velocity range is from $19-24 \mathrm{~km} \mathrm{~s}^{-1}$. The contour levels of each CO molecule are $30,40, \ldots, 90 \%$ of the peak value. Letters A, B, C, and D indicate the different molecular clumps. The dot symbols mark the mapping points. The pink triangle represents the IRAS $19282+1814$ source. The red and green crosses represent the submillimeter sources and exciting star HD 231616, respectively. The gray and color bar is in unit of $\mathrm{MJy} \mathrm{sr}^{-1}$ and $\mathrm{K} \mathrm{km} \mathrm{s}^{-1}$, respectively.

Table 1. Observed parameters of each line for each clump.

\begin{tabular}{|c|c|c|c|c|c|c|c|c|c|c|}
\hline \multirow[t]{2}{*}{ Name } & & \multicolumn{3}{|c|}{${ }^{12} \mathrm{CO} J=1-0$} & \multicolumn{3}{|c|}{${ }^{13} \mathrm{CO} J=1-0$} & \multicolumn{3}{|c|}{$\mathrm{C}^{18} \mathrm{O} J=1-0$} \\
\hline & & $\begin{array}{l}T_{\mathrm{mb}} \\
(\mathrm{K}) \\
\end{array}$ & $\begin{array}{c}F W H M \\
\left(\mathrm{~km} \mathrm{~s}^{-1}\right)\end{array}$ & $\begin{array}{c}V_{\mathrm{LSR}} \\
\left(\mathrm{km} \mathrm{s}^{-1}\right)\end{array}$ & $\begin{array}{l}T_{\mathrm{mb}} \\
(\mathrm{K})\end{array}$ & $\begin{array}{c}F W H M \\
\left(\mathrm{~km} \mathrm{~s}^{-1}\right)\end{array}$ & $\begin{array}{c}V_{\mathrm{LSR}} \\
\left(\mathrm{km} \mathrm{s}^{-1}\right)\end{array}$ & $\begin{array}{l}T_{\mathrm{mb}} \\
(\mathrm{K})\end{array}$ & $\begin{array}{c}F W H M \\
\left(\mathrm{~km} \mathrm{~s}^{-1}\right)\end{array}$ & $\begin{array}{c}V_{\mathrm{LSR}} \\
\left(\mathrm{km} \mathrm{s}^{-1}\right)\end{array}$ \\
\hline Clump A & Main & 33.5 & 3.2 & 23.4 & 18.6 & 2.0 & 23.4 & 2.2 & 1.9 & 23.6 \\
\hline \multirow[t]{2}{*}{ Clump B } & Main & 33.8 & 1.8 & 23.1 & 18.8 & 1.4 & 23.3 & 3.2 & 1.0 & 23.5 \\
\hline & Residual & 11.2 & 2.2 & 25.3 & 7.5 & 1.5 & 25.0 & 2.0 & 1.2 & 25.0 \\
\hline \multirow[t]{2}{*}{ Clump C } & Main & 19.1 & 2.8 & 23.2 & 12.1 & 1.5 & 23.0 & 2.6 & 1.3 & 23.1 \\
\hline & Residual & 4.8 & 1.1 & 25.5 & 2.3 & 1.0 & 24.9 & - & - & - \\
\hline \multirow{2}{*}{ Clump D } & Main & 22.0 & 2.1 & 22.7 & 9.6 & 1.9 & 22.6 & 1.4 & 2.2 & 22.6 \\
\hline & Residual & 10.2 & 1.5 & 24.5 & 3.6 & 0.9 & 24.5 & - & - & - \\
\hline
\end{tabular}

in Fig. 4b, c, and d. In Fig. $4 \mathrm{~b}$ and $\mathrm{d}$, the emission maps of ${ }^{12} \mathrm{CO} J=1-0,{ }^{13} \mathrm{CO} J=1-0$, and $\mathrm{C}^{18} \mathrm{O} J=1-0$ clearly show four clumps. The velocity component of 24 to $27 \mathrm{~km} \mathrm{~s}^{-1}$ is not associated with the clumps A-D in Fig. 4c, but is consistent with the PAH emission of H II region G53.54-0.01. Hence, we suggest that the velocity component of $24-27 \mathrm{~km} \mathrm{~s}^{-1}$ is another cloud clump, which is weaker than the emission of $19-24 \mathrm{~km} \mathrm{~s}^{-1}$, as seen in Table 1 . The velocity component of $24-27 \mathrm{~km} \mathrm{~s}^{-1}$ may be collected into the clumps A, B, C, and D with the expansion of $\mathrm{H}$ II region G53.54-0.01. The $\mathrm{C}^{18} \mathrm{O} J=1-0$ emission is used 

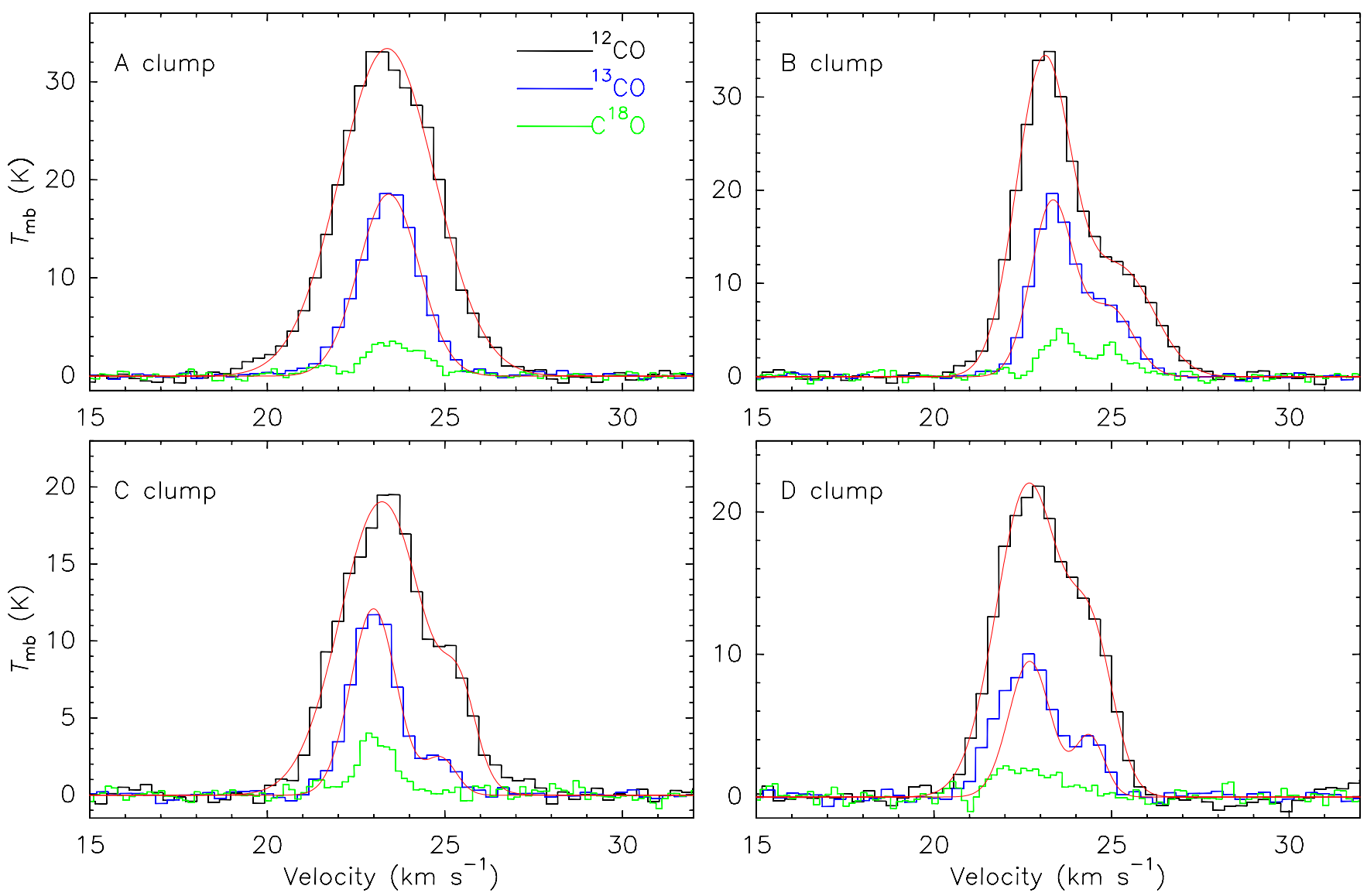

Fig. 5. ${ }^{12} \mathrm{CO} J=1-0,{ }^{13} \mathrm{CO} J=1-0$, and $\mathrm{C}^{18} \mathrm{O} J=1-0$ spectra at the peak position of the molecular cores A, B, C, and D. To better show the profile of $\mathrm{C}^{18} \mathrm{O} J=1-0$ line, only the $\mathrm{C}^{18} \mathrm{O} J=1-0$ intensity is amplified to 1.5 times of the original intensity. The red lines represent the Gaussian fitted lines.

to trace the dense clump. Comparing the $\mathrm{C}^{18} \mathrm{O} J=1-0$ emission in Fig. 4b with that in Fig. 4c, we found that only the position of clump B has the strong $\mathrm{C}^{18} \mathrm{O} J=1-0$ emission with the velocity component of $24-27 \mathrm{~km} \mathrm{~s}^{-1}$, which is responsible for the double-peaks profile of $\mathrm{C}^{18} \mathrm{O} J=1-0$ in clump B. One of peaks at $\sim 23 \mathrm{~km} \mathrm{~s}^{-1}$ is associated with clump B, another at $\sim 25 \mathrm{~km} \mathrm{~s}^{-1}$ may belong to the collected gas.

Anderson et al. (2011) gave that the hydrogen radio recombination line (RRL) velocity is $23.9 \pm 0.3 \mathrm{~km} \mathrm{~s}^{-1}$ for $\mathrm{H}$ II region G53.54-0.01, which are associated with the main systemic velocities of each clump in the $\mathrm{C}^{18} \mathrm{O} J=1-0$ line. According to the Galactic rotation model of Fich et al. (1989) together with $R_{\odot}=8.5 \mathrm{kpc}$ and $V_{\odot}=220 \mathrm{~km} \mathrm{~s}^{-1}$, where $V_{\odot}$ is the circular rotation speed of the Galaxy, we obtain a kinematic distance of 2.0 or 8.1 kpc to H II region G53.54-0.01. Hunter \& Massey (1990) found that $\mathrm{H}$ II region G53.54-0.01 has a photometric distance of $1.7 \mathrm{kpc}$. Hereafter, we will adopt the near kinematic distance of $2.0 \mathrm{kpc}$. Using the optical thin ${ }^{13} \mathrm{CO} J=1-0$ line and assuming local thermodynamical equilibrium (LTE) we estimate the $\mathrm{H}_{2}$ column density towards each clump and the collected gas in Fig. 5. Moreover, we assume that the collected gas has the same excitation temperature as each clump. The column density is determined by Garden et al. (1991)

$N_{{ }^{13} \mathrm{CO}}=4.6 \times 10^{13} \frac{\left(T_{\mathrm{ex}}+0.89\right)}{\exp \left(-5.29 / T_{\mathrm{ex}}\right)} \int T_{\mathrm{mb}} \mathrm{d} v \mathrm{~cm}^{-2}$,

where $\mathrm{d} v$ is the velocity range in $\mathrm{km} \mathrm{s}^{-1}$, and $T_{\text {ex }}$ is the excitation temperature in $\mathrm{K}$. We estimate $T_{\text {ex }}$ following the equation

$T_{\mathrm{ex}}=5.53 / \ln \left[1+5.53 /\left(T_{\mathrm{mb}}+0.82\right)\right]$, where $T_{\mathrm{mb}}$ is the corrected main-beam temperature of ${ }^{12} \mathrm{CO} J=$ $1-0$. Here we use the relation $N_{\mathrm{H}_{2}} / N_{{ }^{3} \mathrm{CO}} \approx 5 \times 10^{5}$ (Simon et al. 2001) and Gaussian fit line parameters to estimate the $\mathrm{H}_{2}$ column density.

If the clumps are approximately spherical in shape, the mean number density of $\mathrm{H}_{2}$ is estimated to be

$n\left(\mathrm{H}_{2}\right)=1.62 \times 10^{-19} N_{\mathrm{H}_{2}} / d$,

where $d$ is the averaged diameter of the clumps in parsecs (pc), measured from Fig. 4.

Moreover, their mass is given by

$M_{\mathrm{H}_{2}}=\frac{1}{6} \pi d^{3} \mu_{\mathrm{g}} m\left(\mathrm{H}_{2}\right) \mathrm{n}\left(\mathrm{H}_{2}\right)$,

where $\mu_{\mathrm{g}}=1.36$ is the mean atomic weight of the gas, and $m\left(\mathrm{H}_{2}\right)$ is the mass of a hydrogen molecule. The obtained results are all listed in Table 2.

\section{Discussion}

\subsection{The dynamics of the large-scale infrared bubble and $H$ II region G53.54-0.01}

The large-scale infrared bubble shows the half-shell structure at $8 \mu \mathrm{m}$, which just surrounds a large radio shell centered at $l=53.9^{\circ}$ and $b=0.2^{\circ}$. Leahy et al. (2008) suggested that the radio shell may belong to SNR G53.9+0.2 at a distance of $\simeq 7.3 \mathrm{kpc}$. The large-scale infrared bubble contains three 
J.-L. Xu and B.-G. Ju: Star formation associated with a large-scale infrared bubble

Table 2. Physical parameters of the clumps in LTE.

\begin{tabular}{cccccccc}
\hline \hline Name & & $\begin{array}{c}T_{\mathrm{ex}} \\
\mathrm{K}\end{array}$ & $\begin{array}{c}d \\
(\mathrm{pc})\end{array}$ & $\begin{array}{c}\int T_{\mathrm{mb}} \mathrm{d} v \\
\left(\mathrm{~K} \mathrm{~km} \mathrm{~s}^{-1}\right)\end{array}$ & $\begin{array}{c}N_{\mathrm{H}_{2}} \\
\left(\mathrm{~cm}^{-2}\right)\end{array}$ & $\begin{array}{c}n\left(\mathrm{H}_{2}\right) \\
\left(\mathrm{cm}^{-3}\right)\end{array}$ & $\begin{array}{c}M \\
\left(M_{\odot}\right)\end{array}$ \\
\hline Clump A & Main & 37.0 & 2.1 & 113.5 & $1.2 \times 10^{23}$ & $9.3 \times 10^{3}$ & $3.0 \times 10^{3}$ \\
Clump B & Main & 37.3 & 3.2 & 65.7 & $6.7 \times 10^{22}$ & $3.4 \times 10^{3}$ & $3.9 \times 10^{3}$ \\
& Residual & & & 25.6 & $2.6 \times 10^{22}$ & $1.3 \times 10^{3}$ & $1.5 \times 10^{3}$ \\
Clump C & Main & 22.6 & 0.9 & 56.5 & $3.9 \times 10^{22}$ & $7.0 \times 10^{3}$ & $1.8 \times 10^{2}$ \\
& Residual & & & 5.6 & $3.8 \times 10^{21}$ & $0.7 \times 10^{3}$ & $0.2 \times 10^{2}$ \\
Clump D & Main & 25.5 & 1.2 & 49.9 & $3.7 \times 10^{22}$ & $5.0 \times 10^{3}$ & $3.5 \times 10^{2}$ \\
& Residual & & & 16.2 & $1.2 \times 10^{22}$ & $1.6 \times 10^{3}$ & $1.0 \times 10^{2}$ \\
\hline
\end{tabular}

H II regions (G53.54-0.01, G53.64+0.24, and G54.09-0.06), while a Crab-like young SNR G54.1+0.3 is situated on the radio shell. Because the kinematic distance of SNR G54.1+0.3 is $\simeq 6.2 \mathrm{kpc}$ from HI and CO observations (Leahy et al. 2008), SNR G54.1+0.3 is not related to the radio shell. Using the GRS data, we found that the large-scale infrared bubble is associated with several ${ }^{13} \mathrm{CO} J=1-0$ clumps. The clumps have two velocity components that peak at $\sim 24$ and $38 \mathrm{~km} \mathrm{~s}^{-1}$. The velocity component peak at $38 \mathrm{~km} \mathrm{~s}^{-1}$ coincides with $\mathrm{H}$ II regions G53.64+0.24 and G54.09-0.06, while that at $24 \mathrm{~km} \mathrm{~s}^{-1}$ is associated with $\mathrm{H}$ II region G53.54-0.01. Hence, the $8 \mu \mathrm{m}$ emission consistent with H II region G53.54-0.01 should belong to the foreground emission, and only overlap with the large-scale infrared bubble in the line of sight. Additionally, the $8 \mu \mathrm{m}$ emission of the inner border is denser in the large-scale infrared bubble. Moreover, the ${ }^{13} \mathrm{CO} J=1-0$ emission related to $\mathrm{H}$ II region G54.09-0.06 shows a comet-like morphology. We conclude that the large-scale infrared bubble is likely to be formed by ultraviolet (UV) radiation from the progenitor star of SNR 53.9+0.2.

In addition, H II region G53.54-0.01 is close to a filamentary molecular cloud. We also made the observations in ${ }^{12} \mathrm{CO} J=1-0,{ }^{13} \mathrm{CO} J=1-0$, and $\mathrm{C}^{18} \mathrm{O} J=1-0$ towards H II region G53.54-0.01 and its adjacent region. Four clumps are found in this region. The profiles of ${ }^{12} \mathrm{CO} J=1-0$ in clump A only show the blue wing. Clump A is associated with IRAS $19282+1814$. Sun et al. (2003) concluded that IRAS $19282+1814$ has a blueshifted monopolar molecular outflow. To confirm the outflow, we made two position-velocity (PV) diagrams (Fig. 6) with the cuts through the peak position of clump A along the north-south and east-west directions in ${ }^{12} \mathrm{CO} \mathrm{J}=$ 1-0 line. Clump A shows the bipolar structure presented by the blue and red dashed arrows. The blueshifted component has obvious velocity gradients with respect to the systemic velocity of clump A. The redshifted component has also the velocity gradient along the inverse direction, but the gradient is not very obvious relative to the blueshifted component. The distributions of redshifted and blueshifted velocity components are an indication of outflow motion. However, the profiles of ${ }^{12} \mathrm{CO} J=1-0$ and ${ }^{13} \mathrm{CO} J=1-0$ in clumps $\mathrm{B}-\mathrm{D}$ are only broadened in red wings. Comparing with the profile of $\mathrm{C}^{18} \mathrm{O} J=1-0$ in clump $\mathrm{B}$, the red wing may not be broadened, but indicate a component. The component may be the collected gas, which expands into the molecular cloud containing clumps A-D, after being collected with the expansion of H II region G53.54-0.01.

\subsection{Triggered star formation}

Koo et al. (2008) detected a star-forming loop around SNR G54.1+0.3. They suggested that the star-forming loop may be triggered by the progenitor star of G54.1+0.3. Because of the selected Class I and Class II sources in a large-scale region, we

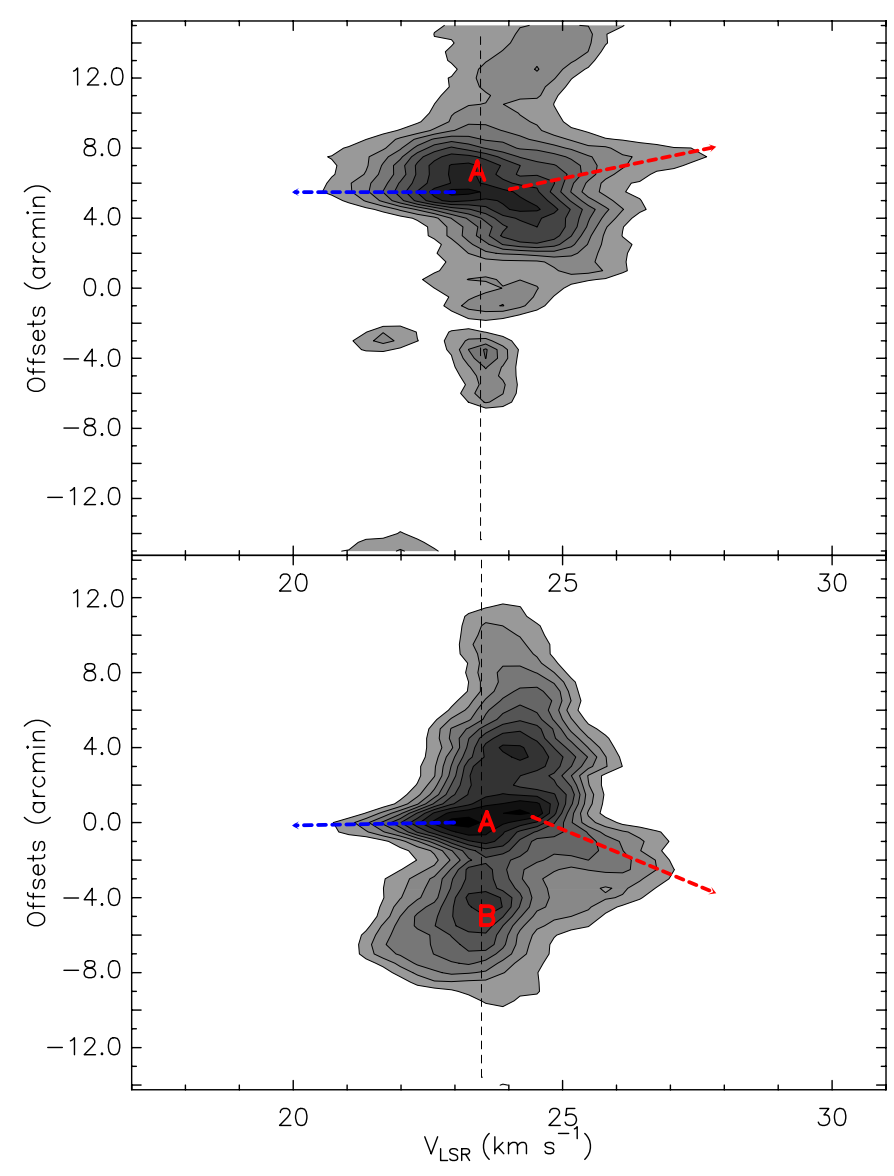

Fig. 6. P-V diagram constructed from ${ }^{12} \mathrm{CO} J=1-0$ transition for clump A. The contour levels are $10,20, \ldots, 90 \%$ of the peak value, with the cut through the peak position of clump A along the east-west (upper panel) and north-south (under panel) directions. The blue and red dashed arrows show the blueshifted and redshifted components of the outflow. The dotted line in the spectra marks the systemic velocity of clump A.

did not find the star-forming loop around SNR G54.1+0.3. Three EGOs sources are found located in the G54.09-0.06 complex, as well as over three H II regions and two small-scale bubbles. This distribution is an indication of an active star-forming region located in the large-scale infrared bubble around SNR G53.9+0.2.

Class I and Class II sources are mostly concentrated around $\mathrm{H}$ II region G53.54-0.01, which is expanding into a filament molecular cloud. Four clumps are found around H II region G53.54-0.01, but only clump A associated with a massive YSO (IRAS 19282+1814) may have an outflow motion. Adopting the angle of $90^{\circ}$ and the clump size as the outflow size, the dynamic timescale of the outflow is estimated roughly by equation 
$t=9.78 \times 10^{5} R / V(\mathrm{yr})$, where $V$ in $\mathrm{km} \mathrm{s}^{-1}$ is the maximum flow velocity relative to the cloud systemic velocity, and $R$ in pc is the outflow size. The average dynamical timescale of the outflow is $4.3 \times 10^{5}$ yr. Clump A has the mass of $3.0 \times 10^{3} M_{\odot}$, indicating a massive star-forming region. The above analysis suggests that the triggered star formation occurred in the region around H II region G53.54-0.01. The dynamical age of H II region G53.540.01 can also be used to decide whether YSOs are triggered by the $\mathrm{H}$ II region. Assuming the emission is optically thin free-free thermal continuum, the ionizing luminosity $Q_{\mathrm{Ly}}$ is computed by Condon (1992) as

$$
Q_{\mathrm{Ly}}=7.54 \times 10^{46}\left(\frac{v}{\mathrm{GHz}}\right)^{0.1}\left(\frac{T_{\mathrm{e}}}{\mathrm{K}}\right)^{-0.45}\left(\frac{S_{v}}{\mathrm{Jy}}\right)\left(\frac{D}{\mathrm{kpc}}\right)^{2} \mathrm{~s}^{-1},
$$

Where $v$ is the frequency of the observation, $S_{v}$ is the observed specific flux density, and $D$ is the distance to the $\mathrm{H}$ II region. Anderson et al. (2011) found that HII region G53.54-0.01 has the flux density of $1.3 \mathrm{Jy}$ at $9 \mathrm{GHz}$. Because the spectral type of the exciting star of $\mathrm{H}$ II region G53.54-0.01 is B0V (Hunter \& Massey 1990), we adopt an electron temperature of $33340 \mathrm{~K}$ (Vacca et al. 1996). Finally, we obtain $Q_{\mathrm{Ly}} \simeq 4.5 \times 10^{45} \mathrm{ph} \mathrm{s}^{-1}$.

Using a simple model described by Dyson \& Williams (1980) and assuming an H II region expanding in a homogeneous medium, we estimate the dynamical age of the $\mathrm{H}$ II region as

$t_{\mathrm{HII}}=7.2 \times 10^{4}\left(\frac{R_{\mathrm{HII}}}{\mathrm{pc}}\right)^{4 / 3}\left(\frac{Q_{\mathrm{Ly}}}{10^{49} \mathrm{ph} \mathrm{s}^{-1}}\right)^{-1 / 4}\left(\frac{n_{\mathrm{i}}}{10^{3} \mathrm{~cm}^{-3}}\right)^{-1 / 2} \mathrm{yr}$,

where $R_{\mathrm{HII}}$ is the radius of the $\mathrm{H}$ II region, $n_{\mathrm{i}}$ is the initial number density of the gas, and $Q_{\mathrm{Ly}}$ is the ionizing luminosity. Adopting the measured radius of $\mathrm{H}$ II region G53.54-0.01 ( $2.3 \mathrm{pc})$ obtained from Fig. 5, and assuming an initial number density of $\sim 10^{3} \mathrm{~cm}^{-3}$, we derive a dynamical age of $1.5 \times 10^{6} \mathrm{yr}$ for $\mathrm{H}$ II region G53.54-0.01.

Comparing the age of $\mathrm{H}$ II region G53.54-0.01 with that of YSOs (Class I and Class II sources) shown on its border, we suggest that these YSOs are likely to be triggered by $\mathrm{H}$ II region G53.54-0.01. Two processes have been considered for the triggering of star formation at the edge of the $\mathrm{H}$ II regions (Deharveng et al. 2010), namely collect and collapse (CC) and radiation driven implosion (RDI). In the $\mathrm{CC}$ process, a compressed layer of high-density neutral material forms between the ionization front and shock front preceding it in the neutral gas, and star formation occurs when this layer becomes gravitationally unstable, which is characterized by the presence of the fragments regularly spaced along the molecular ring or shell. In the RDI process, the shocks drive into pre-existing density structures and compress them to form stars, which is characterized by cometary globules or optically bright rims. The PAH emission of $\mathrm{H}$ II region G53.54-0.01 shows the cometary globule, but we detected the collected gas in clumps B, C, and D. Furthermore, we identified an outflow only in clump A associated with the massive YSO IRAS $19282+1814$, but did not detect the collected gas. We suggest that the collected gas expands into the pre-existing clump A, the local density of clump A increases, then it collapses to be a massive star. The $\mathrm{CC}$ process is responsible for the YSOs and a massive star formation around $\mathrm{H}$ II region G53.54-0.01. This process not only collects the diffuse gas near the pre-existing molecular cloud, but also adds the collected gas into the pre-existing molecular cloud.

\section{Conclusions}

Using the Spitzer-IRAC $8 \mu \mathrm{m}$ and GRS ${ }^{13} \mathrm{CO} J=1-0$ archival data, we have studied a large-scale infrared bubble centered at $l=53.9^{\circ}$ and $b=0.2^{\circ}$. The $\mathrm{H}$ II regions G53.540.01 , G53.64+0.24, and G54.09-0.06 are located on the bubble. Molecular observations in ${ }^{12} \mathrm{CO} J=1-0,{ }^{13} \mathrm{CO} J=1-0$ and $\mathrm{C}^{18} \mathrm{O} J=1-0$ with the PMO $13.7 \mathrm{~m}$ radio telescope were performed to investigate the detailed distribution of molecular material associated with HII region G53.54-0.01 (Sh2-82). The results can be summarized as follows.

1. The large-scale infrared bubble presents a half-shell morphology at $8 \mu \mathrm{m}$. H II regions G54.09-0.06 and G53.64+0.24 have recombination line velocities of $42.1 \pm 0.8 \mathrm{~km} \mathrm{~s}^{-1}$ and $38 \pm 1.8 \mathrm{~km} \mathrm{~s}^{-1}$, respectively, while $23.9 \pm 0.3 \mathrm{~km} \mathrm{~s}^{-1}$ for $\mathrm{H}$ II region G53.54-0.01, hence we concluded that the $8 \mu \mathrm{m}$ emission related to H II region G53.54-0.01 should belong to the foreground emission, and only overlap with the largescale infrared bubble in the line of sight.

2. The large-scale infrared bubble has the dense inner border and several pillar-like structures. Moreover, the ${ }^{13} \mathrm{CO} J=$ 1-0 emission related to H II region G54.09-0.06 shows a comet-like morphology, suggesting that the large-scale infrared bubble is likely to be formed by ultraviolet (UV) radiation from the progenitor star of old SNR 53.9+0.2.

3. Three EGO sources, three H II regions, and two small-scale bubbles (N116 and N117) are found situated at the G54.090.06 complex, suggesting an active massive star-forming region. We suggest that the velocity of N116 and N117 may be $\sim 38 \mathrm{~km} \mathrm{~s}^{-1}$.

4. In the ${ }^{18} \mathrm{CO} J=1-0$ line, we found four cloud clumps on the northeastern border of $\mathrm{H}$ II region G53.54-0.01. Comparing the spectral profiles of ${ }^{12} \mathrm{CO} J=1-0$ and ${ }^{13} \mathrm{CO} J=1-0$ with that of the ${ }^{18} \mathrm{CO} J=1-0$ peak at each clump, we found a collected gas component, which has rushed into three clumps B-D, but not into the clump A.

5. clump A with a mass of $3000 M_{\odot}$ is associated with a submillimeter wavelength continuum source and a massive YSO. Additionally, clump A has an outflow motion. The estimated dynamical timescale of the outflow is $4.3 \times 10^{5} \mathrm{yr}$, indicating a forming massive star. The derived ages of G53.54-0.01 is $1.5 \times 10^{6} \mathrm{yr}$. The significant enhancement of several Class I and Class II YSOs around G53.54-0.01 indicates the presence of some recently formed stars. Taking into account the age of G53.54-0.01 and YSOs, we find that G53.54-0.01 may trigger the formation of these YSOs via the collect-andcollapse process.

Acknowledgements. We are very grateful to the anonymous referee for his/her helpful comments and suggestions. We are also grateful to the staff at the Qinghai Station of PMO for their assistance during the observations. Thanks for the Key Laboratory for Radio Astronomy, CAS, for partly supporting the telescope operation. This work was supported by the National Natural Science Foundation of China (Grant No. 11403042), and was also supported by the young researcher grant of national astronomical observatories, Chinese academy of sciences.

\section{References}

Allen, L. E., Calvet, N., \& D'Alessio, P., 2004, ApJS, 154, 363

Anderson, L. D., Bania, T. M., Balser, D. S., \& Rood, R. T. 2011, ApJ, 194, 32

Camilo, F., Lorimer, D. R., Bhat, N. D. R., et al. 2002, ApJ, 574, L71 
Condon, J. J. 1992, ARA\&A, 30, 575

Condon, J. J., Cotton, W. D, Greisen, E. W., et al., 1998, AJ, 115, 1693

Chen, X., Shen, Z., Li, J., Xu, Y., \& He, J. 2010, ApJ, 710, 150

Churchwell, E., Povich, M. S., Allen, D., et al. 2006, ApJ, 649, 759

Churchwell, E., Watson, D. F., Povich, M. S., et al. 2007, ApJ, 670, 428

Cyganowski, C. J., Whitney, B. A., Holden, E., et al. 2008, AJ, 136, 2391

Deharveng, L., Schuller, F., Anderson, L. D., et al. 2010, A\&A, 523, A6

Dewangan, L. K., \& Ojha, D. K. 2013, MNRAS, 429, 1386

Dewangan, L. K., Ojha, D. K., Anandarao, B. G., Ghosh, S. K., \& Chakraborti, S. 2012, A\&A, 756, A151

Di Francesco, J., Johnstone, D., Kirk, H., MacKenzie, T., \& Ledwosinska, E. 2008, ApJS, 175, 277

Dyson, J. E., \& Williams, D. A. 1980, Physics of the interstellar medium (Manchester University Press)

Fazio, G. G., Hora, J. L., Allen, L. E., et al. 2004, ApJS, 154, 10

Fich, M., Blitz, L., \& Stark, A. A. 1989, ApJ, 342, 272

Garden, R. P., Hayashi, M., Hasegawa, T., et al. 1991, ApJ, 374, 540

He, J. H., Takahashi, S., \& Chen, X. 2012, ApJS, 202, 1

Hunter, D. A., \& Massey, P. 1990, AJ, 99, 846

Jackson, J. M., Rathborne, J. M., Shah, R. Y. et al. 2006, ApJS, 163, 145
Ji, W.-G., Zhou, J.-J., Esimbek, J., et al. 2012, A\&A, 544, A39

Koo, B.-C., McKee, C. F., Lee, J.-J., et al. 2008, ApJS, 673, L147

Leahy, D. A., Tian, W., \& Wang, Q. D. 2008, AJ, 136, 1477

Li, Jun-Yu., Jiang, Zhi-Bo., Liu, Yao., \& Wang, Yuan. 2013, Rev. Astron. Astrophys., 13, 921

Lockman, F. J. 1989, ApJS, 71, 469

Lu, F. J., Wang, Q. D., Aschenbach, B., Durouchoux, P., \& Song, L. M. 2002, ApJS, 568, L49

Pomarès, M., Zavagno, A., Deharveng, L., et al. 2009, A\&A, 494, 987

Sherman, R. A. 2012, ApJ, 760, 58

Simon, R., Jackson, J. M., Clemens, D. P., Bania, T. M., \& Heyer, M. H. 2001, ApJ, 551, 747

Sun, K., \& Wu, Y. 2003, Chin. Astron. Astrophys., 27, 73

Vacca, W. D., Garmany, C. D., \& Shull, J. M. 1996, ApJ, 460, 914

Velusamy, T., \& Becker, R. H. 1988, AJ, 95, 1162

Velusamy, T., Goss, W. M., \& Arnal, E. M. 1986, A\&A, 7, 105

Watson, C., Hanspal, U., \& Mengistu, A. 2010, ApJ, 716, 1478

Yu, N.-P., \& Wang, J.-J. 2012, Rev. Astron. Astrophys., 12, 651

Zhang, C.-P., Wang, J.-J., \& Xu, J.-L. A\&A, 550, 117

Zuo, Y.-X., Yang, J., Shi, S.-C., et al. 2004, Ch. J. Astron. Astrophys., 4, 390 